\title{
Insegnare gli ultimi settant'anni. Una panoramica sui manuali di storia per la scuola secondaria di secondo grado
}

di Carla Marcellini and $\underline{\text { Agnese Portincasa }}$

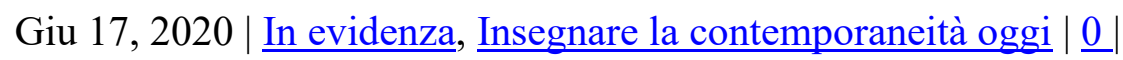

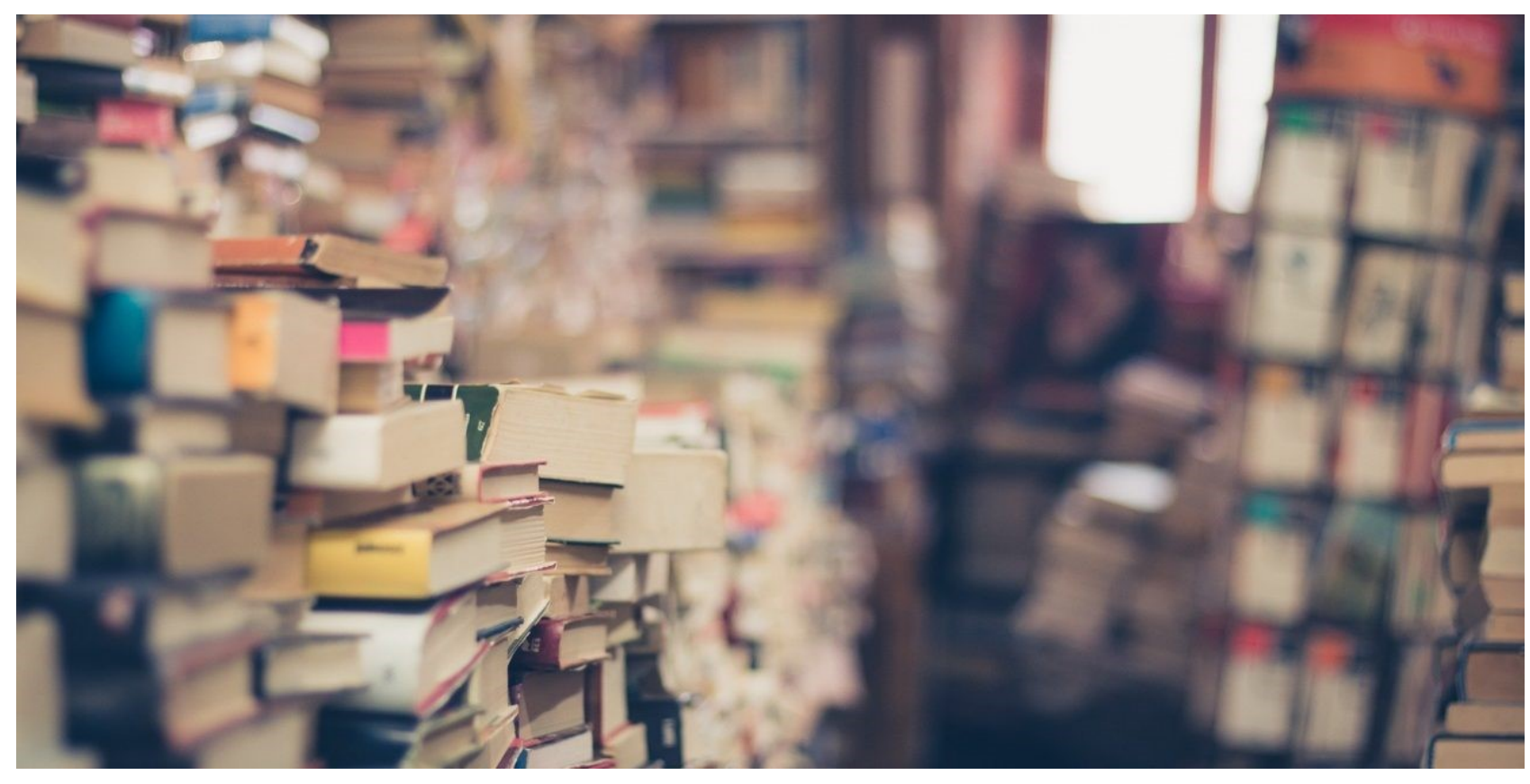

\begin{abstract}
L'articolo riporta i dati della ricerca dedicata all'insegnamento della storia degli ultimi settant'anni, presentata alla prima edizione dei Cantieri della Didattica dell'Istituto Nazionale Ferruccio Parri che si sono tenuti a Rimini nel maggio 2019. Il focus è la manualistica scolastica e l'intento è avviare una riflessione che corre parallelamente agli approfondimenti scientifici sul tema. Nella loro proposta le autrici intendono favorire la riflessione dei docenti sull'utilizzo di questo tradizionale dispositivo per lo studio della storia, nella logica di fornire alcuni suggerimenti utili per la progettazione didattica.
\end{abstract}

- Introduzione

- I manuali

- I caratteri della ricerca

- Tre indicatori: spazio dedicato; struttura e temi; cronologie e proposte di periodizzazione

- Aspetti caratterizzanti e tipologie di manuali

- Conclusioni

Introduzione

La nostra ricerca nasce come contributo per i Cantieri della didattica organizzati dall' Istituto

Nazionale Ferruccio Parri nel maggio 2019; circostanza in cui è stata avviata una riflessione sulla 
manualistica a partire dalle specifiche difficoltà che molti docenti della secondaria di secondo grado manifestano nell'insegnare la storia del secondo Novecento. Difficoltà dovute a una serie di fattori oggettivi e soggettivi di cui occorre tenere conto. La diminuzione delle ore di storia, che risale a una riforma di più di un decennio fa, ha portato le ore annuali del triennio a sessantasei anche per i licei, uniformando il monte ore a quello degli istituti tecnici. L'unica eccezione è il liceo classico che continua ad avere novantanove ore annuali di storia.

A fronte di tale limite oggettivo, persiste la percezione che la storia del secondo Novecento sia una parte del programma in cui si accavallano moltissimi fatti, tanti protagonisti, una dimensione globale delle questioni storiche - che rende complicato tenere insieme le tre scale spaziali (Italia, Europa, Mondo) - tanti argomenti e problemi che spaziano dalla storia politica a quella sociale, da quella economica a quella culturale in senso lato. Sembra difficile orientarsi per trovare il filo di un gomitolo aggrovigliato di storie attorno alle quali sedimentare conoscenze, interpretazioni, riflessioni che, attraverso la costruzione di un percorso didattico fattibile ed esauriente, permetta allo studente di acquisire competenze storiche e di cittadinanza al termine del secondo ciclo di istruzione.

Andrebbe notato come non sia così per altri periodi della storia insegnata che rientrano nei programmi scolastici. Nell'ambito della storia antica, di quella medievale o moderna, ad esempio, la stragrande maggioranza dei docenti si uniforma ad alcune categorie di analisi del passato che sono entrate in maniera stabile nella manualistica. Sembra, insomma, che gli insegnanti si sentano più a loro agio quando si tratta di affrontare epoche che meglio rispondono a convenzioni storiche o manualistiche consolidate.

Ci siamo allora domandate: è possibile che una delle difficoltà riscontrate dai docenti nel trattare la storia del recente passato possa derivare, almeno in parte, da come è trattata nei libri di testo?

Avviare un'analisi su quanto si trova nei manuali scolastici e su come sono strutturati ci è sembrata una strada utile per iniziare a comprendere come vengono insegnati gli ultimi settanta anni di storia e per capire quale contributo l'Istituto Nazionale Ferruccio Parri e la rete nazionale degli Istituti storici della Resistenza possano offrire alla scuola.

\section{I manuali}

I manuali di storia presenti sul mercato sono numerosissimi, in media ogni casa editrice ne ha parecchi in catalogo: diversi per edizioni e autori. Si tratta di prodotti molto elaborati e ricchi di informazioni, con un nucleo di contenuti piuttosto statico, ma rinnovati frequentemente dal punto di vista editoriale e grafico. Negli ultimi anni gli aggiornamenti sono stati determinati, oltre che da esigenze di mercato, anche dal rinnovamento della prima prova dell'Esame di stato. L'abolizione del tema di storia (tipologia $\mathrm{C}$ ) e la sua inclusione nella tipologia $\mathrm{B}$, hanno determinato la necessità di modificare in corsa gli apparati didattici.

Parlando in termini generali il manuale è un testo complesso in cui gli eventi del passato sono sistematizzati e collocati entro la dimensione spaziale e temporale, spiegati e interpretati. La conoscenza di come tale strumento sia strutturato e di come le informazioni siano organizzate e distribuite al suo interno ne facilita l'uso. Saper individuare l'interpretazione storiografica e l'organizzazione tematica, oltre che cronologica e spaziale, aiuta il docente a selezionare i contenuti e a organizzare un lavoro differenziato in base alle priorità della propria programmazione. È vero, però, che un'analisi tanto meticolosa è fuori portata per la maggior parte dei docenti che si trovano 
nell'impossibilità di dedicare il tempo che sarebbe necessario alla comparazione di prodotti tutto sommato simili, in un panorama editoriale ricchissimo di offerta.

Il senso di questa ricerca nasce, dunque, dall'esigenza di contribuire a una riflessione bottom up di carattere operativo, utile anche a proporre semplici operazioni di prima analisi su una serie di manuali differenti. L'obiettivo non è, naturalmente, quello di valutarne la qualità, ma di cercare di descriverne le caratteristiche peculiari ai fini del loro utilizzo nella pratica scolastica quotidiana. Il risultato presentato è da considerarsi come la messa a fuoco delle problematiche più evidenti: spunto per ulteriori riflessioni a venire.

\section{I caratteri della ricerca}

Il primo obiettivo metodologico è consistito nel rendere il più possibile omogeneo e comparabile il campione. Sono stati presi in esame sette manuali per le classi quinte della secondaria di secondo grado, licei e istituti tecnici, tralasciando quelli per gli istituti professionali che hanno scansioni cronologiche differenti.

Sempre per motivi di uniformità del campione abbiamo deciso di escludere la versione digitale dei singoli manuali: prodotti che appaiono molto diversi gli uni dagli altri e quindi ben difficilmente comparabili. Si passa da piattaforme articolate e interattive, ricche di esercizi e materiali didattici, di testi storiografici e repertori di immagini, a espansioni digitali che offrono poco di più della versione in pdf del testo cartaceo. Senza contare che molti docenti non utilizzano le espansioni digitali, anche perché l'accesso alle piattaforme richiede registrazioni, uso di password e l'attivazione di una procedura che spesso non si esaurisce in pochi secondi, come la pratica didattica richiederebbe. ${ }^{[1]}$

Nella ricca offerta di manualistica abbiamo selezionato le opere prendendo in considerazione due semplici criteri:

- l'anno di edizione (nella logica di privilegiare i manuali più recenti e aggiornati);

- la diffusione (nella logica di comparare prodotti che godono di un sicuro apprezzamento e della fiducia da parte dei docenti).

Fra i manuali più recenti e di maggiore diffusione abbiamo analizzato:

1. V. Castronovo, Impronta storica 3, La Nuova Italia, Firenze, 2017.

2. G. De Luna, M. Meriggi, La rete del tempo 3. Il Novecento e gli anni Duemila, PearsonParavia, Milano-Torino, 2018.

3. G. Borgognone, D. Carpanetto, L'idea della storia 3, Bruno Mondadori, Milano 2017.

4. A. Barbero, C. Frugoni, C. Sclarandis, La storia. Progettare il futuro 3. Il Novecento e l'età attuale, Zanichelli, Bologna, 2019.

5. S. Luzzatto, G. Alonge, Dalle storie alla storia 3. Dal Novecento ad oggi, Zanichelli, Bologna, 2016.

6. M. Bresciani, P. Palmieri, M. Rovinello, F. Violante, Storie. Il passato nel presente 3., Giunti-Treccani, Milano, 2019.

7. G. Gentile, L. Ronga, A. Rossi, Erodoto magazine 3. Novecento e l'inizio del XXI secolo, Editrice La scuola, Brescia, 2017. 
Per favorire un'analisi coerente ed efficace abbiamo scelto di lavorare focalizzandoci su due livelli: da un lato entrare nella struttura del volume ci ha permesso di comprendere quanto spazio è dedicato alla seconda metà del Novecento, quali siano le principali tematizzazioni, quali periodizzazioni siano utilizzate e quali cronologie siano evidenziate; dall'altro abbiamo ricercato elementi comuni, similarità strutturali, aspetti caratterizzanti per provare a raggruppare i testi in alcune tipologie.

Attorno alla struttura abbiamo analizzato le sezioni e i capitoli per individuare l'arco cronologico trattato, lo spazio a esso dedicato, la rilevanza di tematizzazioni e cronologie.

Per fare emergere gli elementi comuni abbiamo scelto di individuare un tema periodizzante che coprisse un arco temporale vasto e uno spazio geografico che consentisse di lavorare su una scala spaziale ampia. La scelta è ricaduta sulla guerra fredda: nucleo tematico fondante per la lettura delle vicende del secondo dopoguerra fino al 1989, oltre che categoria storiografica senz'altro consolidata di cui i docenti dispongono per spiegare il secondo Novecento. Attraverso la trattazione dedicata a tale tema abbiamo cercato di intercettare gli sguardi e gli approcci che ogni progetto editoriale riversava sugli ultimi settanta anni di storia. Dall'analisi abbiamo rilevato quelli che, a nostro parere, sono quattro differenti modelli di manualistica: alcuni manuali ci sono parsi più "tradizionali", soprattutto in contrapposizione ad altri più "innovativi"; alcuni ci sono parsi più "complessi" e "onnicomprensivi", soprattutto quando contrapposti ad altri volutamente più "semplificati" o "dalla parte degli studenti".

Nell'analisi abbiamo posto attenzione anche alle scelte lessicali e terminologiche degli autori, nella convinzione che le parole rivelino spesso l'impostazione storiografica, il modello narrativo ed esplicativo.

Per brevità i manuali saranno, da qui in avanti, indicati con il solo cognome del primo autore in copertina.

\section{Tre indicatori: spazio dedicato; struttura e temi; cronologie e proposte di periodizzazione}

\section{Lo spazio dedicato: il numero di pagine}

Una prima, banale, osservazione è che i volumi presi in esame sono tutti tomi consistenti. È un dato che non coglie di sorpresa nessuno, docenti e studenti sanno per esperienza che la manualistica dedicata alla storia è difficilmente snella. E tuttavia andrebbe notato come, nel caso di queste opere, i contenuti trattino del solo Ventesimo secolo (se si pensa al triennio della secondaria di secondo grado, il primo e il secondo volume raccolgono e descrivono, nella medesima mole, porzioni ben più ampie di vicende storiche). Già questo primo indizio conferma come, avvicinandosi al presente, la manualistica storica selezioni sempre meno e tende a far prevalere la dimensione evenemenziale.

Passando ai numeri osserviamo che i manuali più poderosi - Barbero e Borgognone - superano le ottocento pagine: si tratta di un dato interessante perché, come vedremo, non è questa l'unica affinità fra i due testi. Più contenuto è il numero di pagine per Bresciani e per Castronovo fino ad arrivare ai manuali più snelli: dalle 666 pagine di Luzzatto alle 642 di Gentile. Il dato di Gentile va commisurato, però, alla scelta di un formato editoriale piuttosto grande - e inusuale nella manualistica - che, come suggerito nel titolo, dovrebbe ricordare un magazine. 


\section{Lo spazio dedicato Numero di pagine}

\begin{tabular}{cccc}
\hline Manuale & Pagine (da-a) & Complessivo & $\begin{array}{c}\text { In proporzione } \\
\text { rispetto al } \\
\text { volume }\end{array}$ \\
\hline Barbero & Da 467-832 & 365 & $43 \%$ \\
\hline Borgognone & Da 562-832 & 270 & $32 \%$ \\
\hline Bresciani & Da 398 a 760 & 362 & $45 \%$ \\
\hline Castronovo & Da 414-650 & 236 & $36 \%$ \\
\hline De Luna & Da 380-644 & 264 & $40 \%$ \\
\hline Gentile & Da 361-642 & 281 & $43 \%$ \\
\hline Luzzatto & Da 347-666 & 319 & $47 \%$ \\
\hline
\end{tabular}

Tabella 1: Lo spazio dedicato alla trattazione degli ultimi settant'anni di storia

Oltre il dato più genericamente numerico va notato che i manuali non dedicano al secondo Novecento la medesima estensione: calcolando in termini percentuali si osserva come, ad esempio, manuali con minor numero di pagine gli dedichino il $47 \%$ del complessivo, mentre altri più poderosi si limitino a un 32\% (Tabella 1). Pur restando a un puro dato di superficie lo spazio dedicato aiuta a rivelare - nei singoli progetti editoriali e autoriali - le scelte di una differente trattazione degli argomenti.

Interessante osservare inoltre che, nonostante tutti i manuali contemplino una sezione per la stretta attualità - l'inizio del XXI secolo, fino al 2016 - in nessuno prevale quantitativamente la storia dell'ultimo settantennio: la seconda parte del Novecento e gli anni più recenti sono, dunque, genericamente meno frequentati. La manualistica, insomma, rispecchia certe consuetudini didattiche diffuse o forse, da una differente angolazione, le modella.

\section{Struttura e temi: unità e capitoli}

Approfondendo l'analisi della struttura possiamo rilevare quante unità o sezioni sono dedicate al secondo Novecento, oltre che da quanti capitoli ognuna di esse è composta (Tabella 2). 


\section{Unità e capitoli La struttura}

\begin{tabular}{cccc}
\hline Manuale & Sezioni/Unità & $\begin{array}{c}\text { Capitoli per } \\
\text { sezione/unità }\end{array}$ & $\begin{array}{c}\text { Capitoli in } \\
\text { totale }\end{array}$ \\
\hline Barbero & 3 & $5+2+2$ & 9 \\
\hline Borgognone & 2 & $5+2$ & 7 \\
\hline Bresciani & 2 & $4+5$ & 9 \\
\hline Castronovo & 2 & $5+2$ & 7 \\
\hline De Luna & $3(+1)$ & $3+2+2$ & 7 \\
\hline Gentile & 8 & $1+1+1+1+1+1+1+1$ & 8 \\
\hline Luzzatto & 3 & $2+2+2$ & 6 \\
\hline
\end{tabular}

Tabella 2: Unità e capitoli dedicati alla trattazione degli ultimi settant'anni di storia

La struttura più semplificata è quella adottata da Gentile, nel quale la tematizzazione corrisponde a un capitolo che è contemporaneamente un'unità. Barbero, De Luna e Luzzatto dedicano tre unità al secondo Novecento, tutti gli altri manuali due.

La struttura di Luzzatto appare la più simmetrica; la sistematizzazione degli argomenti è volutamente improntata a un certo equilibrio evidente anche nelle scelte contenutistiche: ogni sezione prevede una cornice che coincide, come vedremo, con una biografia di riferimento. Borgognone e Castronovo operano una scelta che genera la percezione di uno sbilanciamento: cinque capitoli sistematizzano la stragrande maggioranza dei contenuti; due, invece, sono dedicati al mondo globale (Borgognone) o alla storia d'Italia (Castronovo).

Uno sguardo agli indici delle singole unità suggerisce altre considerazioni. Per Luzzatto si conferma una precisa scelta di ordinamento: le tre sezioni contemplano sempre due capitoli ciascuna e il secondo è sempre dedicato all'Italia. Nelle intenzioni dei curatori è evidente il desiderio di offrire un modello ricorrente di narrazione che connota l'analisi e rassicura il lettore.

Puntando l'attenzione sul termine guerra fredda vediamo che Barbero, Castronovo, De Luna e Gentile usano espressamente la locuzione, anche se con scelte di titolazione che evidenziano tagli interpretativi differenti (in Castronovo, ad esempio, guerra fredda entra in dialogo con il termine "multipolarismo"). Bresciani e Luzzatto, invece, non ne fanno menzione. Nella terza unità di Bresciani si parla di un "lungo dopoguerra"; nella quarta di "difficoltà dell'Occidente e dell'ascesa di nuovi mondi". Borgognone preferisce il termine "bipolarismo". 
Per quanto riguarda la storia del tempo presente, Barbero, Borgognone e Luzzatto gli dedicano espressamente una sezione, mentre De Luna preferisce introdurre un contenitore extra-sistema intitolato Lo storico di fronte alle sfide del presente. Sempre parlando della più stretta attualità che coincide grosso modo con il primo ventennio del XXI secolo - le scelte descrittive e lessicali mostrano un'ardua consonanza terminologico-concettuale: Barbero parla di "mondo multipolare", Borgognone introduce la locuzione di "era globale" che sarebbe l'evoluzione di un "mondo postbipolare". La varietà terminologica traccia la sostanza di una difficoltà oggettiva, peraltro del tutto comprensibile, nell'inquadrare in modo didatticamente efficace la storia degli ultimi vent'anni. Molto differenti sono le scelte circa la storia nazionale. Luzzatto, come abbiamo visto, è l'unico a trattare la storia d'Italia in ogni unità, mentre Borgognone, De Luna e Castronovo gli dedicano la sezione conclusiva. Anche Barbero la isola, ma dedicandogli uno spazio mediano.

Una più chiara lettura dell'analisi e della comparazione si può leggere nelle schede seguenti nelle quali sono sinteticamente raccolti, per ciascun manuale: ${ }^{[2]}$

- $\quad$ i titoli di unità/sezioni (con le relative cronologie);

- i titoli dei capitoli;

- le proposte di periodizzazione (attraverso l'indicazione della presenza di linee del tempo e dei nodi evento che le costituiscono). 


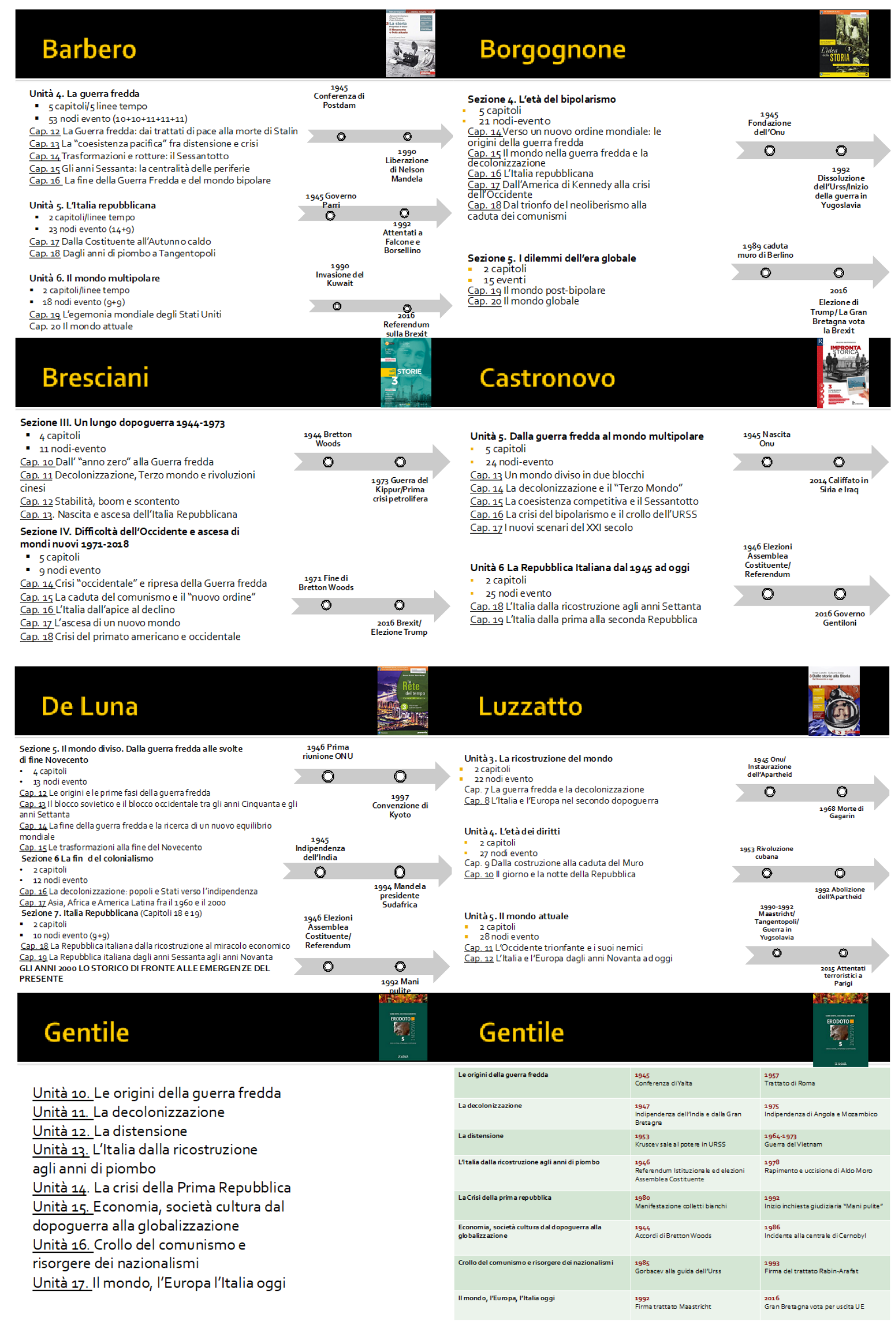




\section{Scarica le schede singole in una cartella .zip}

\section{Cronologie e proposte di periodizzazione}

Le schede hanno il pregio di sintetizzare molti dati interessanti, non ultimi le scelte di cronologia e periodizzazione. La difformità più evidente riguarda Gentile che opta per strumenti semplificati e, nel caso della periodizzazione, "non tradizionali". È, infatti, l'unico manuale che non utilizza linea del tempo. In alternativa ogni capitolo è introdotto da una tabella a tre colonne nella quale sei nodievento sono posti al centro, preceduti da un "prima" a sinistra e seguiti da un "dopo" a destra. Si tratta di una modalità che certo contribuisce a scardinare certi effetti di evenemenzialità pura che inducono spesso lo studente a introiettare semplici automatismi di memorizzazione. Il rischio, però, è quello di facilitare interpretazioni favorevoli a un nesso strettissimo e poco problematico fra causa ed effetto. Insomma per fuggire da una zona di confort si finisce per correre di rischio di cadere in un'altra, concettualmente più pericolosa.

E tuttavia la questione che Gentile inquadra con la propria scelta non è di poco conto: le linee del tempo sono strumenti utilissimi per l'apprendimento della storia, ma possono essere come i falsi amici che chi studia le lingue ben conosce. Si dà per scontato che la rappresentazione grafico/sequenziale favorisca la comprensione, ma spesso quello che accade - in assenza di un supporto da parte del docente che potrebbe, ad esempio, guidare a un laboratorio che miri a costruirle - è favorire una semplice memorizzazione di cifre volatili.

Tutti gli altri manuali usano le linee del tempo: alcuni in una pagina iniziale introduttiva di sezione (Borgognone, De Luna, Luzzatto), altri all'inizio di ogni capitolo (Barbero, Bresciani, Castronovo). Il loro utilizzo non è sempre identico, soprattutto nei termini della quantità dei nodi-evento: Barbero ne indica complessivamente 94, Luzzatto 77, Castronovo 49, Borgognone 36 e De Luna 35, Bresciani 20 (è evidente che nello scarto fra le 94 date di Barbero e le 20 di Bresciani ci sia una difformità che implica una precisa scelta di rilevanze).

Passando alle date-evento periodizzanti - poste agli estremi di una data linea del tempo - abbiamo notato che tre manuali partono dal 1945 indicando l'anno come quello di fondazione dell'ONU (Borgognone, Castronovo, Luzzatto). Luzzatto aggiunge si tratti anche dell'anno d'instaurazione dell'apartheid (filo che riprenderà segnalando il 1992 come anno della sua abolizione e atto conclusivo dell'unità dal titolo L'età dei diritti). De Luna slitta di un anno avanti e indica con precisione la prima riunione dell'Onu. Barbero segnala la Conferenza di Potsdam, Gentile quella di Yalta; Bresciani gli accordi di Bretton Woods.

Quanto alla data-evento che chiude il periodo della stretta contemporaneità: Barbero, Borgognone, Bresciani e Gentile identificano la Brexit (in alcuni casi accompagnata dall'elezione di Donald Trump negli Stati Uniti). Luzzatto anticipa di un anno e stabilisce un punto fermo negli attentati terroristici a Parigi; Castronovo di due per risalire al Califfato in Siria e Iraq. De Luna si differenzia e fissa l'ultima data periodizzante nel XX secolo: 1997, l'anno della Convenzione di Kyoto.

Vi sono altri eventi che stabiliscono gerarchie periodizzanti che possiamo definire non usuali. La guerra fredda di Barbero termina con la liberazione di Mandela nel 1990, mentre la sua presidenza, nel 1994, segna la fine del colonialismo per De Luna. I dilemmi dell'era globale iniziano per Borgognone con la caduta del muro di Berlino; Il lungo dopoguerra di Bresciani termina con la crisi petrolifera; Gentile introduce una questione ambientale pressoché inesistente altrove, indicando l'incidente di Chernobyl del 1986 come data periodizzante; la morte di Jurij Gagarin, personaggio simbolo della ricostruzione del mondo dopo la Seconda guerra mondiale, chiude un ciclo, e il personaggio si merita la copertina nel volume di Luzzatto. 
Dietro l'ampio ventaglio delle date proposte si nascondono certamente precise e differenti interpretazioni storiografiche, ma emerge anche una scelta di autorialità di cui il docente che utilizza il manuale dovrebbe avere percezione.

Aspetti caratterizzanti e tipologie di manuali

\section{I manuali "tradizionali": Castronovo e De Luna}

I manuali che abbiamo definito di impianto tradizionale sono nuove edizioni di opere realizzate alcuni anni fa. Essi hanno aspetti comuni che li caratterizzano. La guerra fredda è la categoria storiografica attraverso la quale è costruita l'unità di lavoro che va dal 1945 agli anni Novanta. All'interno dell'unità dedicata il racconto ha la medesima struttura narrativa: il bipolarismo (o mondo diviso) è seguito dalla sua crisi che confluisce per Castronovo in un "mondo multipolare" di "nuovi scenari" e per De Luna in "svolte" o "trasformazioni" di "fine Novecento". La fine del mondo bipolare, dunque, coincide con la fine della periodizzazione che gli autori adottano per organizzare e concettualizzare il secondo Novecento.

Dopo la fine del mondo bipolare le chiavi interpretative paiono indebolirsi. Non a caso, alla fine del volume, si trovano dei capitoli-contenitore in cui sono affrontati temi e problemi del presente che non sono entrati nella periodizzazione proposta. La dimensione fattuale, legata alla vicinanza temporale, prevale su quella interpretativa, dando la chiara sensazione - molto probabilmente voluta - si tratti di questioni di attualità più che di storia.

La storia italiana è trattata separatamente: strategia che alcuni insegnanti trovano utile perché offre la possibilità di lavorare isolatamente sul secondo dopoguerra nazionale. La differenza più evidente fra le due opere, invece, è che mentre De Luna separa le questioni dell'Africa, dell'Asia e dell'America latina trattandole nella sezione dedicata alla decolonizzazione, Castronovo le inquadra all'interno della sezione sulla guerra fredda.

Si tratta di manuali molto strutturati difficili da smontare o comporre in funzione della programmazione docente. Un solo esempio per chiarire: volendo spiegare la guerra fredda trattando contemporaneamente dell'Italia, dei trattati di pace o semplicemente della questione di Trieste o volendo inserire il boom economico italiano nell'espansione economica dell'Età dell'oro, l'insegnante è costretto a spostarsi da una sezione all'altra; ciò che è, di per sé, fonte di sicuro spaesamento per gli studenti.

\section{I manuali "innovativi": Luzzatto e Bresciani}

Nei manuali definiti di tipo innovativo appare evidente la volontà di costruire un prodotto nuovo sia per quanto riguarda la periodizzazione che per la tematizzazione. Mentre Luzzatto individua tre periodizzazioni (1945-1961 - La ricostruzione del mondo; 1953-1992 - L'età dei diritti; 1990-2015 - Il mondo attuale), in Bresciani il secondo Novecento si organizza in due periodi (1944-1973 - Il lungo dopoguerra e 1971-2018 - Difficoltà dell'occidente e ascesa di mondi nuovi).

La guerra fredda non serve tanto a periodizzare quanto a leggere il dato storico: i manuali si occupano per intero degli ultimi settanta anni, e tutto ciò che entra nel testo è letto come storia del presente e non attualità. Le tre scale spaziali sono in continuo dialogo: la storia d'Italia si intreccia con quella europea e mondiale. 
Anche la tematizzazione va oltre lo schema tradizionale. In Luzzatto il dopoguerra non è raccontato in termini di blocchi contrapposti, ma con lo sguardo positivo di un mondo che si ricostruisce ( $\mathrm{La}$ ricostruzione del mondo), a tal punto da poter chiamare l'unità successiva $L$ 'età dei diritti. In Bresciani la fine della guerra fredda e la caduta del muro (all'interno della sezione Difficoltà dell'Occidente e ascesa di nuovi mondi), non rappresentano la fine del mondo bipolare, ma l'inizio di una fase di transizione e trasformazione che si apre su due scenari: da un lato la globalizzazione e dall'altro il declino dell'Occidente.

È interessante rimarcare due espedienti narrativi che enfatizzano l'intento innovativo di tali due proposte. Luzzatto usa la storia di un personaggio per ogni sezione: Jurij Gagarin per La ricostruzione del mondo, Nelson Mandela per L'età dei diritti, Malala Yousafzai per Il mondo attuale. Si tratta di una scelta che pone l'attenzione sul valore storico della soggettività: la collocazione - in apertura - ha l'evidente funzione didattica di suggerire come si possa entrare nella Storia a partire dalle storie individuali (come il titolo Dalle storie alla Storia suggerisce).

Bresciani fa una scelta diversa, anche questa non tradizionale: esplicitare la struttura interpretativa solitamente nascosta tra le righe -, nella logica di rendere più facilmente accessibili le categorie storiografiche di cui si avvale il testo per costruire la narrazione storica. A questo servono le Parole della storiografia poste in esordio di ogni sezione: "Guerra fredda", "Terzo mondo", "Trent'anni gloriosi", "Repubblica dei partiti" per Il lungo dopoguerra. "Neoliberismo", "Rivoluzioni/transizioni", "Crisi dell'antifascismo", "Globalizzazione", "Secolo americano" per Difficoltà dell'occidente e ascesa di mondi nuovi.

\section{I manuali "onnicomprensivi": Borgognone e Barbero}

Le abbiamo definite opere "onnicomprensive" perché più delle altre richiamano il modello della saggistica specialistica, soprattutto per quanto riguarda le scelte lessicali e l'impianto narrativo. Sono, inoltre, manuali che moltiplicano stimoli e focalizzazioni, proposti attraverso il ricorso a immagini, tabelle, parole chiave, concettualizzazioni. Barbero, ad esempio, conclude le sezioni proponendo precisi percorsi di approfondimento attraverso scelte di luoghi, di opere letterarie o cinematografiche (non sfugge il riferimento ad alcune scelte del palinsesto di Rai Storia, probabilmente per tracciare percorsi famigliari ai docenti). Si tratta, inoltre, di manuali che si avvalgono di ricchissimi apparati di fonti, lemmari disciplinari, proposte di laboratorio e di esercizi, storiografia. Nel Borgognone una lunga sezione è dedicata a Il secolo breve di Eric Hobsbawm: diversi estratti di uno dei più noti classici della storiografia del Novecento sono estrapolati per offrirli alla riflessione degli studenti.

Manuali molto versatili e capaci di offrire spunti per le più svariate personalizzazioni di percorso, richiedono competenze sicure da parte del docente che deve sapere selezionare con precisione. Un paio di esempi per comprendere: nel capitolo quattordicesimo, Verso un nuovo ordine mondiale: le origini della guerra fredda, Borgognone propone le seguenti Parole concetto: "Superpotenze"; "Bipolarismo"; "Onu"; "Tribunale Militare Internazionale", "Cortina di ferro"; "Contenimento"; "Guerra fredda"; "Piano Marshall"; "Comecon"; "Crisi di Berlino"; "Nato"; "Patto di Varsavia". Anche se è vero che si tratta di intenderle come parole-chiave utili ad approfondire il discorso, la specificità della scelta terminologica chiama in causa competenze di lettura un poco fuori portata per gli studenti cui è rivolto il testo. Senza contare che i termini richiamano contesti distinti: quello politico, economico, giudiziario, quello istituzionale e militare.

Le tematizzazioni che Barbero introduce nel testo come Nuclei fondanti hanno, invece, lo svantaggio di confondere il piano della lettura gerarchica proposta nelle focalizzazioni suggerite 
nella paragrafazione: anche in questo caso la moltiplicazione delle chiavi di accesso ai contenuti complica la lettura di dati e percorsi.

Nelle due opere le proposte di intreccio sono numerose e quanto mai stimolanti, ma spesso agiscono su livelli differenti e richiamano numerose e specifiche inferenze.

Le pagine sono dense, con fitti espedienti grafici a introdurre contenuti extra-testuali. Per tutti questi motivi si tratta di testi cui è indispensabile una mediazione didattica costante: manuali che con una certa difficoltà si può pensare possano essere affidati allo studio individuale degli studenti che potrebbero sentirsi frustrati nell'ordinare tanta complessità.

\section{I manuali "dalla parte dello studente": Gentile}

Già descritta come opera che si distingue per scelta di semplificazione, l'abbiamo definita "dalla parte dello studente". Già nel titolo l'utilizzo del termine magazine indica una strategia accattivante che fa l'occhiolino a certe grafiche da rivista patinata: con molte immagini fotografiche a tutta pagina, in grande formato e una struttura che ricorda i blog.

Lo stile è sicuramente divulgativo e presenta scelte lessicali dalle quali traspare un niente affatto banale lavoro di traduzione/trasposizione a uso dello studente. La messa a fuoco è sui processi, a enfatizzare una logica di comprensione della storia nella quale prevale, più che l'acquisizione di conoscenze disciplinari, una finalità di educazione alla cittadinanza. Lo studio del passato è utile a comprendere meglio il presente: tutto ciò che può definirsi accessorio rispetto a tale obiettivo diventa trascurabile.

L'effetto per il docente può essere straniante: accade che, di fronte a temi sui quali la storiografia ha prodotto tanto, i contenuti lascino insondato qualche interrogativo; ma, al contrario, attorno ad argomenti ostici, spesso poco frequentati proprio a causa della loro complessità (un caso fra tutti: la decolonizzazione) il manuale si rivela provvidenzialmente affidabile, soprattutto per i docenti che hanno in uso la metodologia della flipped classroom.

A ben vedere anche in questo caso la mediazione didattica deve essere costante, persino più avvertita che per i manuali “onnicomprensivi”. Per ragioni che possiamo definire opposte, il docente deve lavorare con gli studenti per evitare che alcune opportune strategie di appianamento narrativo e concettuale non inducano a trascurare una parte della necessaria problematizzazione.

\section{Conclusioni}

Nelle premesse abbiamo avanzato alcune riflessioni sull'insegnamento della storia recente.

La prima e più importante riguarda un fatto facilmente osservabile: la storia del secondo Novecento è poco insegnata e, a distanza di quasi un secolo, i maturandi affrontano l'Esame di stato con conoscenze che spesso s'interrompono all'inizio della guerra fredda. A fronte di questo silenzio chi lavora a scuola coglie spesso negli studenti un vivo e costante interesse per la storia più recente: un desiderio di approfondire che finisce spesso per essere esaudito entro gli spazi del discorso pubblico; quando non addirittura attraverso l'opinione comune, il "sentito dire", oppure attraverso un sapere soggettivo e strumentale filtrato dalla memoria pubblica, privata, familiare. 
Trattare della storia più recente potrebbe, invece, rappresentare una strategia efficace per mettere ragazzi e ragazze nella condizione di pensare il loro presente in una prospettiva storica.

Ma se davvero stiamo descrivendo un'urgenza - e una necessità -, perché si coglie una resistenza a superarla? Che cosa impedisce ai docenti di lavorare in questa direzione?

Volendo tralasciare nel presente contributo le difficoltà legate alla formazione disciplinare dei docenti di storia e l'annosa questione - accennata in apertura - della riduzione del monte ore, la nostra analisi ha mirato a comprendere se tra le difficoltà possa annoverarsi anche il modo in cui la storia recente è trattata nei manuali.

L'analisi ha mostrato che le opere prese in esame aprono a un vario panorama. Si tratta di manuali ricchissimi di stimoli che tendono tutti, anche se con sfumature diverse, a un certo sovraccarico informativo che rischia di mettere in difficoltà l'insegnante il quale fatica a scegliere fra quanto deve finire per apparirgli ugualmente rilevante.

Il primo problema riguarda la mole stessa dei manuali. È chiaro che nessun insegnante può pensare di far studiare ai propri studenti in un solo anno scolastico - con 66 ore a disposizione - libri di seicento, settecento o ottocento pagine. Se è bello poter pensare che un docente abbia davvero il tempo di selezionare anno per anno i contenuti del programma in base alla classe e agli obiettivi che vi si pone (chi sta a scuola sa che una tale operazione non può essere fatta una volta sola, per sempre e per tutte le classi), tuttavia non sempre è un'attività praticabile in maniera costante per l'intero anno scolastico. Insomma se un professionista coscienzioso ha senza dubbio il compito di selezionare, appare comunque auspicabile iniziare a riflettere attorno a una manualistica nella quale si possa lavorare con meno affanno: uno strumento più agile, anche per lo studente.

Restando alle scelte e agli imbarazzi che pone, bisognerebbe considerare come $i$ fatti più recenti entrino nella memoria personale e soggettiva dello stesso insegnante che difficilmente può mostrare quella capacità di lettura critica che possiede nel trattare, tanto per fare un esempio, delle campagne napoleoniche. Manca lo sguardo lungo sul passato e il consolidamento che esso permette nella strutturazione delle buone pratiche. Senza contare, poi, che il curricolo di storia si costruisce spesso attraverso la scelta di argomenti, rilevanze e snodi temporali didatticamente funzionali al programma dell'anno successivo. E invece per la storia degli ultimi decenni non esiste alcuna vicenda successiva da inquadrare per finalità strettamente curricolari. E forse la medesima difficoltà può valere per gli autori di manuali i quali affollano di evenemenziale gli ultimi capitoli dedicati al Novecento, forse per lasciare ai docenti la valutazione su quale sia la cosa migliore da fare.

Se le difficoltà sono molte, la tentazione non può essere di evitarle, fermandosi prima. Al contrario: la consapevolezza di avere per il passato recente chiavi interpretative provvisorie e in progress dovrebbe consentirci di uscire dalla visione un po' distorta che del passato si possa storicamente parlare solo possedendo interpretazioni certe e definitive che derivano dal sedimentarsi di molti metri lineari di letteratura dedicata. La storia degli ultimi venti o trent'anni non è necessariamente un caos di fatti narrati senza gerarchie logiche. Esiste un livello intermedio di lavoro disciplinare che permette al docente di insegnare storia attraverso la lettura di un passato così vicino da essere urgentemente condizionato dalle domande del presente. Domande che sono nella nostra testa, nella testa dei nostri studenti, nella relazione che ne scaturisce e che è la sostanza stessa dell'apprendimento. 
Note:

[1] Sull'offerta digitale abbinata ai manuali cartacei sarebbe più che opportuno avviare un'analisi comparativa che valuti la quantità, la qualità, la ricaduta didattica e non da ultimo la effettiva utilizzabilità dei materiali; ricerca che potrebbe dare molte indicazioni, soprattutto in funzione della didattica a distanza attivata nel corso dell'emergenza Coronavirus.

${ }^{[2]}$ Per recuperare i medesimi parametri e rendere comparabili i dati, il manuale di Gentile ha richiesto una differente schedatura. 